\title{
New behavioral protocols to extend our knowledge of rodent object recognition memory
}

\author{
Mathieu M. Albasser, ${ }^{1}$ Rosanna J. Chapman, Eman Amin, Mihaela D. Iordanova, \\ Seralynne D. Vann, and John P. Aggleton \\ School of Psychology, Cardiff University, Cardiff, CF10 3AT Wales, United Kingdom
}

\begin{abstract}
Animals often show an innate preference for novelty. This preference facilitates spontaneous exploration tasks of novelty discrimination (recognition memory). In response to limitations with standard spontaneous object recognition procedures for rodents, a new task ("bow-tie maze") was devised. This task combines features of delayed nonmatching-to-sample with spontaneous exploration. The present study explored aspects of object recognition in the bow-tie maze not amenable to standard procedures. Two rat strains (Lister Hooded, Dark Agouti) displayed very reliable object recognition in both the light and dark, with the Lister Hooded strain showing superior performance (Experiment 1). These findings reveal the potential contribution of tactile and odor cues in object recognition. As the bow-tie maze task permits multiple trials within a session, it was possible to derive forgetting curves both within-session and between-sessions (Experiment 1). In Experiment 2, rats with hippocampal or fornix lesions performed at normal levels on the basic version of the recognition task, contrasting with the marked deficits previously seen after perirhinal cortex lesions. Next, the training protocol was adapted (Experiment 3), and this modified version was used successfully with mice (Experiment 4). The overall findings demonstrate the efficacy of this new behavioral task and advance our understanding of object recognition.
\end{abstract}

[Supplemental material is available online at http://www.learnmem.org.]

Understanding the neural basis of recognition memory, the ability to discriminate whether a stimulus is novel or familiar, is heavily reliant on animal research. Here, advances have been closely tied to the introduction of new behavioral tests. The preeminent example concerns one-trial tests of recognition memory for monkeys using delayed nonmatching-to-sample (Mishkin and Delacour 1975). These tasks reward the natural preference that monkeys have for selecting novel items and permit multiple recognition trials within a single session. These features make the task relatively easy to train and then maximize findings from small group sizes. Although rat tasks closely based on delayed nonmatching-to-sample have been devised (Aggleton 1985; Mumby et al. 1990; Steckler et al. 1998; Prusky et al. 2004), they are very rarely employed as they are difficult to train and performance levels are unreliable.

Almost all studies of rodent recognition memory now employ the spontaneous object recognition test and its direct variants (Ennaceur and Delacour 1988; Dix and Aggleton 1999; Winters et al. 2008). These tasks again take advantage of an innate preference for novel items, but this preference is displayed by spending more time exploring novel than familiar stimuli. In the standard version of the task, a rodent (rat or mouse) is placed in an arena containing two identical objects and then freely allowed to explore these objects for several minutes ("sample" phase). After a delay, the rodent is placed back in the arena ("test" phase), which now contains one familiar object (a copy of the sample phase objects) and a novel object. Recognition is signified by greater exploration of the novel object. Because the task measures spontaneous behavior, it requires minimal pretraining, but for the same reason it is prone to considerable variance. Unlike

'Corresponding author.

E-mail AlbasserM@cf.ac.uk; fax 0044-2920874858.

Article is online at http://www.learnmem.org/cgi/doi/10.1101//m.1879610. delayed nonmatching-to-sample, each trial (sample plus test phase) takes many minutes, and so only one recognition trial is normally given per session. Advantages are that proactive interference between objects is minimized, and the one-trial design lends itself to episodic-like tests of memory (Dere et al. 2005; Good et al. 2007). Disadvantages include the fact that data accumulation, with appropriate counterbalancing, is slow.

To address these limitations, a new object recognition test using a "bow-tie maze" (Fig. 1A) has been developed for rats (Albasser et al. 2010). This test combines features of delayed nonmatching-to-sample with spontaneous object preference: It permits multiple trials per session, but the measure of recognition comes from the preferential exploration of novelty. The rat is first placed in one end of a bow-tie-shaped maze that contains a single object (object A; Fig. 1B). After a minute, the rat is allowed to run to the other end of the maze where there are two dissimilar objects (A and B; Fig. 1B). Object A is familiar as it is identical to the object previously explored, while object $B$ is novel. Consequently, a rat will typically prefer to explore object $\mathrm{B}$. On the next trial, a minute later, the rat shuttles back to the initial start point, but this time encounters objects B and C. Object B is now familiar, while object $\mathrm{C}$ is novel. The next trial, $1 \mathrm{~min}$ later, is between object $\mathrm{C}$ (now familiar) and object D (novel), and so on. A food reward placed under every object promotes shuttling back and forth within the maze, and encourages interaction with the objects. Unlike delayed nonmatching-to-sample, the food reward is not contingent on first selecting the novel object.

The present study used the bow-tie maze to explore recognition memory on four fronts. In Experiment 1, recognition in the light and recognition in the dark were compared to help determine the cues available to detect object familiarity. It is known that rats can perform recognition tasks when solely reliant on visual cues (Aggleton 1985; Bartko et al. 2007; Winters and Reid 2010), but object recognition based on other modalities remains 

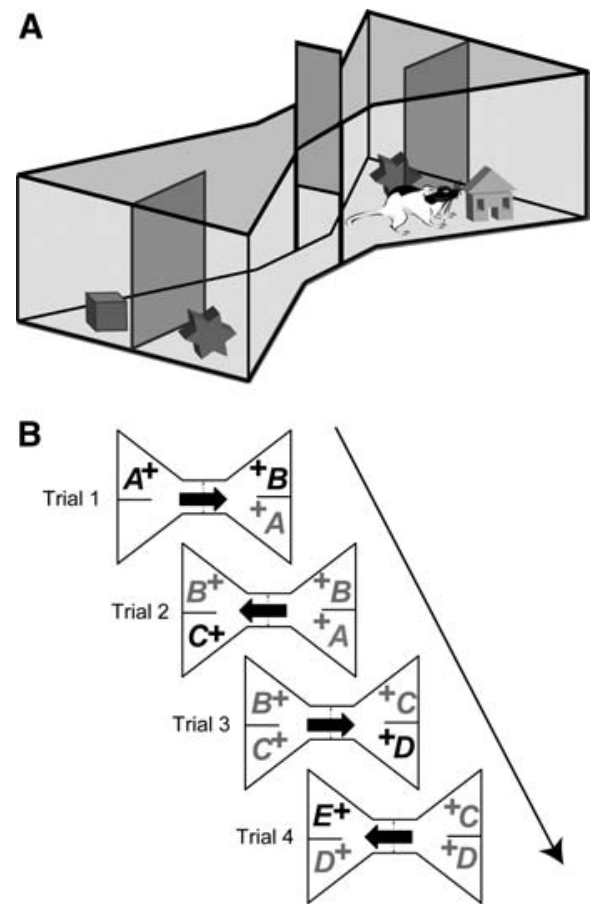

Figure 1. (A) Schematic of the bow-tie maze. A sliding door separates the two ends of the maze in which two objects are placed. $(B)$ General procedure showing the presentation order of the objects in the standard object recognition task. All objects are rewarded $(+)$. (Arrow) Rat movements. (Black print) Novel objects, (gray print) familiar objects.

largely unexplored (Winters and Reid 2010). Potential cues for recognition include odor differences and tactile information. It is known that rats can discriminate novel from familiar olfactory cues (Otto and Eichenbaum 1992; Kesner et al. 2002; Fortin et al. 2004; Wolff et al. 2006), while tactile (e.g., vibrissae) cues can be used to distinguish surfaces (e.g., Birrell and Brown 2000). The bow-tie maze is ideal for studying object recognition in the dark as the rats are rewarded for visiting items in set locations (to receive food rewards), and so should readily approach the objects. In contrast, running the standard spontaneous object recognition test (in an arena) in the dark would be problematic as it is not clear how the rats would first appreciate the presence of the tobe-discriminated objects.

An additional goal of Experiment 1 was to determine how readily the bow-tie maze could be used to compile within-session and between-session forgetting curves. A limitation with the standard spontaneous object task is that with only one trial per session it can be very time consuming to create forgetting curves, while within-session forgetting curves for individual animals are not feasible. These shortcomings create limitations when examining manipulations thought to affect memory. The possibility of deriving within-session forgetting curves is particularly appealing as: (1) it minimized the impact of those factors that introduce variance when performance is compared across sessions, and (2) the animal need not be removed from the maze, which could additionally disrupt performance, e.g., by increasing stress. A further component of Experiment 1 manipulated object memory strength by presenting objects either once ("single") or six times ("repeated"). Recognition was tested after a 3-h delay with the twin goals of determining whether repeated presentation would aid performance and whether these performance levels would be sufficiently above chance so that they could be used to examine factors involved in longer term memory.
At the same time, Experiment 1 provided the opportunity to compare two rat strains, Dark Agouti (DA) and Lister Hooded (LH). Previous studies suggest that the Dark Agouti strain might be particularly good at visual recognition tasks (Aggleton 1996), though others have argued that this strain has aberrant behavioral properties, including higher anxiety and higher levels of inappropriate nonspatial behaviors in spatial learning tasks (Mechan et al. 2002; Harker and Whishaw 2004; but see Aggleton and Vann 2004).

Experiment 2 examined the ability of rats with either hippocampal or fornix lesions to perform object recognition in the bowtie maze. There is a longstanding debate over the impact of hippocampal damage on recognition memory, with mixed findings coming from spontaneous object recognition tests (Clark et al. 2000; Mumby 2001; Gaskin et al. 2003; Winters et al. 2008). The bow-tie maze should prove informative as numerous trials can be run to assess the impact of selective brain lesions. Although in this initial study only short retention delays were examined, these same delays and conditions are highly sensitive to perirhinal cortex lesions (Aggleton et al. 2010; Horne et al. 2010), a brain region regarded as vital for recognition memory (Brown and Aggleton 2001). In Experiment 3 the training protocol changed so that objects did not cover food rewards. Rather, a single food reward was always placed between the test objects. This modification was examined because: (1) it would preclude any exploration scores that were simply derived from attempts to move the test objects in order to uncover the food reward, and (2) it would introduce a task variant that might be amenable to small rodents not able to move objects. Accordingly, Experiment 4 examined the performance of mice (strain $\mathrm{C} 57 \mathrm{Bl} / 6$ ) on a test of object recognition based on the modified version of the bow-tie maze from Experiment 3.

\section{Results}

\section{Experiment 1: Lister Hooded (LH) rats versus Dark Agouti (DA) rats}

In Stage 1, the two rat strains were tested in the light, then the dark, and finally back in the light. All testing used the standard procedure and so all retention delays were $<1 \mathrm{~min}$. In Stages 2 and 3 the retention delays were extended from $<1$ min to $24 \mathrm{~h}$. Two measures of recognition, D1 and D2 (see Materials and Methods, Analysis of behavior), were calculated (Ennaceur and Delacour 1988). D1 is the exploration time for the novel object minus the exploration time for the familiar object. The "cumulative D1" is the sum of D1 scores across trials. D2 is the ratio between the D1 score and the total amount of exploration given to both novel and familiar objects. The $\mathrm{D} 2$ ratio can vary between +1 and -1 , with a positive ratio showing a preference for novelty. An "updated D2" was derived by recalculating the D2 score after each trial within a session (see Materials and Methods).

\section{Pretraining}

An immediate strain difference appeared, as the Dark Agouti group took twice as many sessions (DA rats 14 sessions, LH rats seven sessions) to complete pretraining, i.e., run back and forth to push objects at both ends of the maze to reach food rewards.

\section{Stage 1: Standard object recognition: Light versus dark (delay $<1 \mathrm{~min}$ )}

For the three sessions (light, dark, light), comparisons between the two rat strains were analyzed using $t$-tests (two-tailed). To verify whether animals were performing above chance (zero), D1 and D2 scores were compared against zero, using a one-sample $t$-test (one-tailed). 
Light. The first session (20 trials) tested object recognition in the light (Table 1A; Fig. 2, top). Both LH and DA rats showed a preference for exploring the novel compared with the familiar object as shown by the cumulative D1 (one-sample $t$-test; LH: $t_{(11)}=10.96$, $P<0.001$; DA: $t_{(11)}=14.85, P<0.001$ ) and the updated D2 (onesample $t$-test; LH: $t_{(11)}=14.07, P<0.001$; DA: $t_{(11)}=14.56, P<$ $0.001)$. The $\mathrm{LH}$ rats achieved higher levels of discrimination than the DA rats ( $t$-test; cumulative D1: $t_{(22)}=4.08, P<0.001$; updated D2: $\left.t_{(22)}=2.51, P=0.02\right)$, and the total exploration times (novel plus familiar) were greater for the LH strain $\left(t_{(22)}=\right.$ 2.73, $P=0.012$ ).

Dark. Despite being tested in the dark (Fig. 2, middle), both strains discriminated the novel objects (one-sample $t$-test; cumulative D1: $\mathrm{LH}, \quad t_{(11)}=9.00, P<0.001 ; \quad \mathrm{DA}, t_{(11)}=6.17, P<0.001$; updated D2: LH, $t_{(11)}=11.05, P<0.001 ; \mathrm{DA}, t_{(11)}=7.88, P<$ $0.001)$. Again, the LH strain was superior ( $t$-test; cumulative D1: $t_{(22)}=2.69, \quad P=0.013$; updated D2: $\left.t_{(22)}=3.70, \quad P<0.001\right)$, though both strains spent similar total times exploring the objects $\left(t_{(22)}=0.34, P=0.734\right)$.

Light. Both groups preferred the novel objects (Fig. 2, bottom) as shown by the cumulative D1 (one-sample $t$-test; LH: $t_{(11)}=9.07$, $P<0.001$; DA: $t_{(11)}=9.83, P<0.001$ ) and the updated D2 (onesample $t$-test; LH: $t_{(11)}=13.45, P<0.001$; DA: $t_{(11)}=15.93, P<$ $0.001)$. In this light session, the two strains showed similar levels of discrimination (cumulative D1: $t_{(22)}=1.30, P=0.206$; updated D2: $\left.t_{(22)}=0.024, P=0.98\right)$ and no differences in total exploration times $\left(t_{(22)}=1.81, P=0.083\right)$.

Light versus dark. Direct comparisons of the two strains, involving a one between-group (strain) and a one within-group (light versus dark) ANOVA, used the final D1 and D2 scores (for the light condition the mean score from the two light sessions was used). Rats performed better in the light than the dark (D1: $F_{(1,22)}=38.09$, $\left.P<0.001 ; \mathrm{D} 2: F_{(1,22)}=25.71, P<0.001\right)$, while the overall strain difference reflected the superior recognition scores of the LH rats (D1: $\left.F_{(1,22)}=16.42, P=0.001 ; \mathrm{D} 2: F_{(1,22)}=14.83, P=0.001\right)$. There was no strain $\times$ lighting condition effect (D1: $F<1$; D2:
$F_{(1,22)}=4.04, P=0.057$ ). Total exploration levels (novel plus familiar objects) were higher in the light $\left(F_{(1,22)}=6.83, P=0.016\right)$, and there was a strain $\times$ lighting condition interaction $\left(F_{(1,22)}=\right.$ $8.32, P=0.009)$, as the LH rats spent more time exploring the objects than the DA rats in the light, but not in the dark (simple effects; light: $F_{(1,44)}=8.30, P=0.006$; dark: $F<1$ ).

Effects of proactive interference. Because each session involved 20 trials, there is the possibility of a within-session build of proactive interference that might diminish recognition scores for later trials. This possibility was tested by comparing the combined data (D2) from Trials 1 and 2 (low proactive interference) with the combined data (D2) from Trials 19 and 20 (high proactive interference). The results from both light sessions (i.e., Sessions 1 and 3) were aggregated to give mean D2 scores. Clear evidence that discrimination was reduced in the later trials $\left(F_{(1,22)}=17.88, P<0.001\right)$, consistent with a build up of proactive interference within a session of 20 trials, was found in an ANOVA with one between-group (strain) and one within-group (high versus low proactive interference trials) factor. The lack of a strain by trial interaction $\left(F_{(1,22)}=\right.$ $2.32, P=0.14)$ indicated that no particular strain was more susceptible to proactive interference.

\section{Stage 2: Object recognition: Retention delays of $1 \mathrm{~min}$ to $24 \mathrm{~h}$}

The trials in the first half of each Stage 2 session had retention intervals of $<1 \mathrm{~min}$ as these trials provided initial sample exposures and baseline measures of recognition. Rats were then challenged with novel objects (Table 1B) opposed with objects that had been presented 2-24 min (Short Delay), $3 \mathrm{~h}$ (Mid Delay), or $24 \mathrm{~h}$ (Long Delay) before. As the delay increases, the task should become more difficult as the "familiar" item is forgotten, and so is increasingly treated as "novel." For the Mid Delay and Long Delay conditions, the results for the initial 10 trials $(<1 \mathrm{~min})$ were compared with results from $3 \mathrm{~h}$ and $24 \mathrm{~h}$ delays, respectively, to give two sets of forgetting curves. For the Short Delays (2$24 \mathrm{~min}$ ), the data from the initial 12 trials ( $<1 \mathrm{~min}$ ) were kept separate as the immediately later blocks of trials differed not just in their retention intervals but also the amounts of proactive interference.

Table 1. Presentation order of objects in the different behavioral protocols

(A) Standard object recognition, delay $<1 \min (\operatorname{Exp} 1-$ Stage 1, Exp 2, Exp 3 and 4)

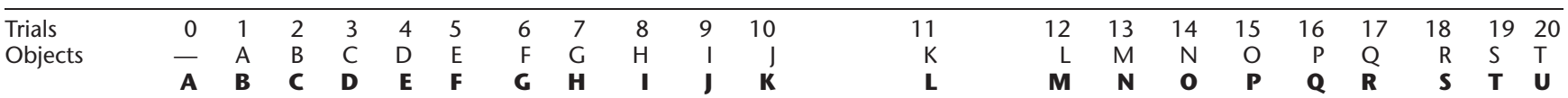

(B) Retention delays from $1 \mathrm{~min}$ to $24 \mathrm{~h}$ (Exp 1-stage 2)

\begin{tabular}{|c|c|c|c|c|c|c|c|c|c|c|c|c|c|c|c|c|c|c|c|c|c|c|}
\hline \multirow{3}{*}{$\begin{array}{l}\text { Trials } \\
\text { Objects }\end{array}$} & \multicolumn{11}{|c|}{$<1 \min$} & & \multicolumn{10}{|c|}{ Short, Mid, or Long retention } \\
\hline & 0 & 1 & 2 & 3 & 4 & 5 & 6 & 7 & 8 & 9 & 10 & & 11 & 12 & 13 & 14 & 15 & 16 & 17 & 18 & 19 & \\
\hline & - & A & $B$ & C & $\mathrm{D}$ & $E$ & $\mathrm{~F}$ & G & $\mathrm{H}$ & 1 & $J$ & Delay & J & 1 & $\mathrm{H}$ & G & $\mathrm{F}$ & $E$ & D & C & B & A \\
\hline
\end{tabular}

(C) Improving levels of object recognition after a 3-h retention interval (Exp 1-stage 3)

\begin{tabular}{|c|c|c|c|c|c|c|c|c|c|c|c|c|c|c|c|c|c|c|c|c|c|c|c|}
\hline \multirow[b]{2}{*}{ Trials } & \multicolumn{14}{|c|}{ Sample phase } & & \multicolumn{8}{|c|}{ Delay phase } \\
\hline & 1 & 2 & 3 & 4 & 5 & 6 & 7 & 8 & 9 & 10 & 11 & 12 & 13 & 14 & & 1 & 2 & 3 & 4 & 5 & 6 & 7 & 8 \\
\hline Objects & G & D & A & D & A & C & B & A & C & D & G & $\mathbf{E}$ & A & G & 3-h delay & $\mathbf{z}$ & $\mathbf{Y}$ & $\mathbf{x}$ & $\mathbf{w}$ & $\mathbf{v}$ & $\mathbf{U}$ & $\mathbf{T}$ & $\mathbf{S}$ \\
\hline & A & C & C & G & G & D & $\mathbf{F}$ & G & D & A & C & H & C & D & & A & B & C & D & $\mathrm{E}$ & $\mathrm{F}$ & G & $\mathrm{H}$ \\
\hline
\end{tabular}

Letters represent novel (black and bold) and familiar (gray) objects. In the last experiment (C), objects that were presented only once ("single") have a gray background. 


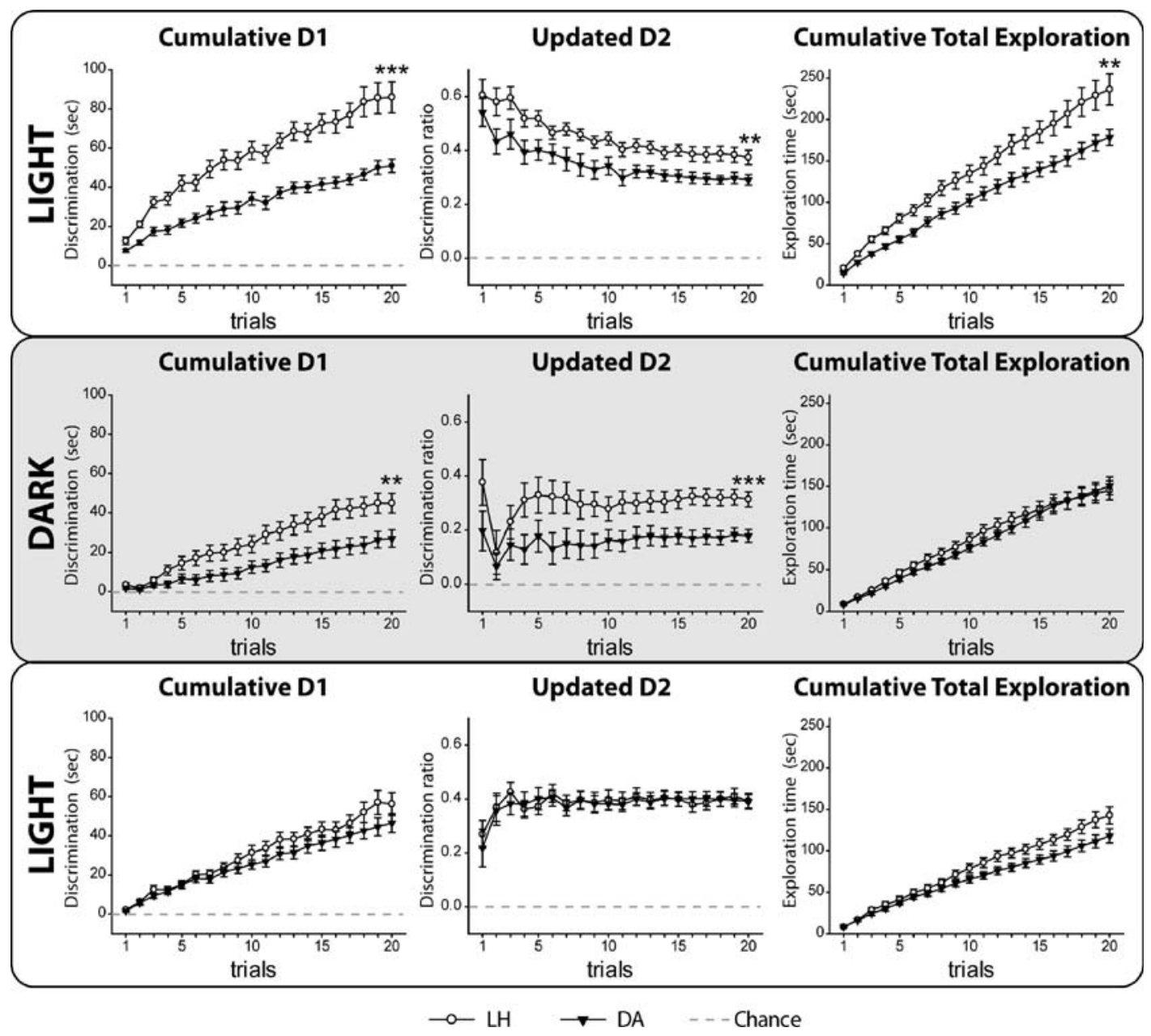

Figure 2. Experiment 1, Stage 1, object recognition in light and dark ( $<1 \mathrm{~min}$ retention interval). Graphs depict mean performance in the light (top), dark (middle), and light (bottom): (left) cumulative D1 score; (middle) updated D2 ratio; (right) cumulative total exploration for all objects. (Black symbols) Dark Agouti rats (DA), (white symbols) Lister Hooded rats (LH). For D1 and D2 scores, a score of zero reflects a failure to discriminate novel from familiar (chance). (Vertical bars) Standard error of the mean (although when small, they are obscured by the symbols). Group differences: (**) $P<0.01,\left({ }^{* *}\right) P<0.001$.

\section{Retention delays of 1-24 min (Short Delays, 24 trials)}

Less than 1 min (Trials 1-12). Both strains preferred the novel objects (Fig. 3, top; one-sample $t$-test; cumulative D1: LH, $t_{(11)}=$ 9.97, $P<0.001$; DA, $t_{(11)}=11.57, P<0.001$; updated D2: $\mathrm{LH}$, $\left.t_{(11)}=13.47, P<0.001 ; \mathrm{DA}, t_{(11)}=18.84, P<0.001\right)$. There was no strain difference ( $t$-test; cumulative D1: $t_{(22)}=0.58, P=0.57$; updated D2: $t_{(22)}=0.49, P=0.63$; total exploration: $t_{(22)}=0.28$, $P=0.78)$.

Two-24 min retention (Trials 13-24). Retention intervals increased by 2 min for every trial after Trial 12, e.g., a 2-min interval for Trial 13, a 12-min interval for Trial 18, and a 24-min interval for Trial 24. The data were, therefore, blocked into three groups of four trials (Trials $13-16,17-20,21-24$ ), with respective mean retention intervals of 5, 13, and 21 min (Fig. 3, top). The two strains and the three retention delays (mean delays of 5, 13, and $21 \mathrm{~min}$ ) were analyzed using a one between $\times$ one within-subject ANOVA. Lister Hooded rats had superior recognition scores (cumulative D1: $F_{(1,22)}=10.21, P=0.004$; updated D2: $F_{(1,22)}=15.61$, $P<0.001$ ). There was a significant effect of delay (cumulative D1: $F_{(2,44)}=10.24, P<0.001$; updated D2: $\left.F_{(2,44)}=5.44, P=0.008\right)$ but no strain $\times$ delay interaction (cumulative D1: $F_{(2,44)}=2.08$, $P=0.138$; updated D2: $F<1$ ).
Both strains of rat were still above chance after the longest (21-min) delays (Fig. 3, top; one-sample $t$-test; cumulative D1: LH, $t_{(11)}=8.70, P<0.001 ;$ DA, $t_{(11)}=2.42, P=0.017$; updated D2: $\left.\mathrm{LH}, t_{(11)}=8.99, P<0.001 ; \quad \mathrm{DA}, t_{(11)}=2.37, P=0.018\right)$. Strain differences for the cumulative D1 scores (simple effects) were only found for the middle delay block $\left(5 \mathrm{~min}: F_{(1,66)}<1\right.$; $13 \mathrm{~min}: F_{(1,66)}=11.89, \quad P<0.001 ; 21 \mathrm{~min}: F_{(1,66)}=1.86, \quad P=$ $0.178)$, though for the updated D2 scores both of the longer delays showed strain differences (simple effects; 5 min: $F_{(1,66)}=3.19, P=$ 0.079 ; 13 min: $F_{(1,66)}=6.87, P=0.011 ; 21 \mathrm{~min}: F_{(1,66)}=7.28, P=$ $0.009)$. In all cases the LH rats were superior. Total object exploration showed no strain difference $(F<1)$.

Retention delays of $3 \mathrm{~h}$ (Mid Delay, 20 trials). Analyses of the 3-h retention delay used a one between-group (LH and DA strains) by one within-group $(<1 \mathrm{~min}$ and $3 \mathrm{~h}$ ) ANOVA. Comparisons of performance (Fig. 3, middle) for $<1 \mathrm{~min}$ (Trials $1-10$ ) versus $3 \mathrm{~h}$ (Trials 11-20; Table 1B) retention intervals found a marked delay effect (D1: $F_{(1,22)}=82.57, P<0.001 ; \mathrm{D} 2: F_{(1,22)}=245.9, P<$ 0.001 ). There was no overall strain difference (D1: $F<1$; D2: $F<$ $1)$ and no strain $\times$ delay interaction $\left(\mathrm{D} 1: F_{(1,22)}=1.86, P=0.19\right.$; D2: $F<1)$. Both the $\mathrm{LH}$ and DA rats still distinguished the novel objects after the 3-h delay (one-sample $t$-test; cumulative 


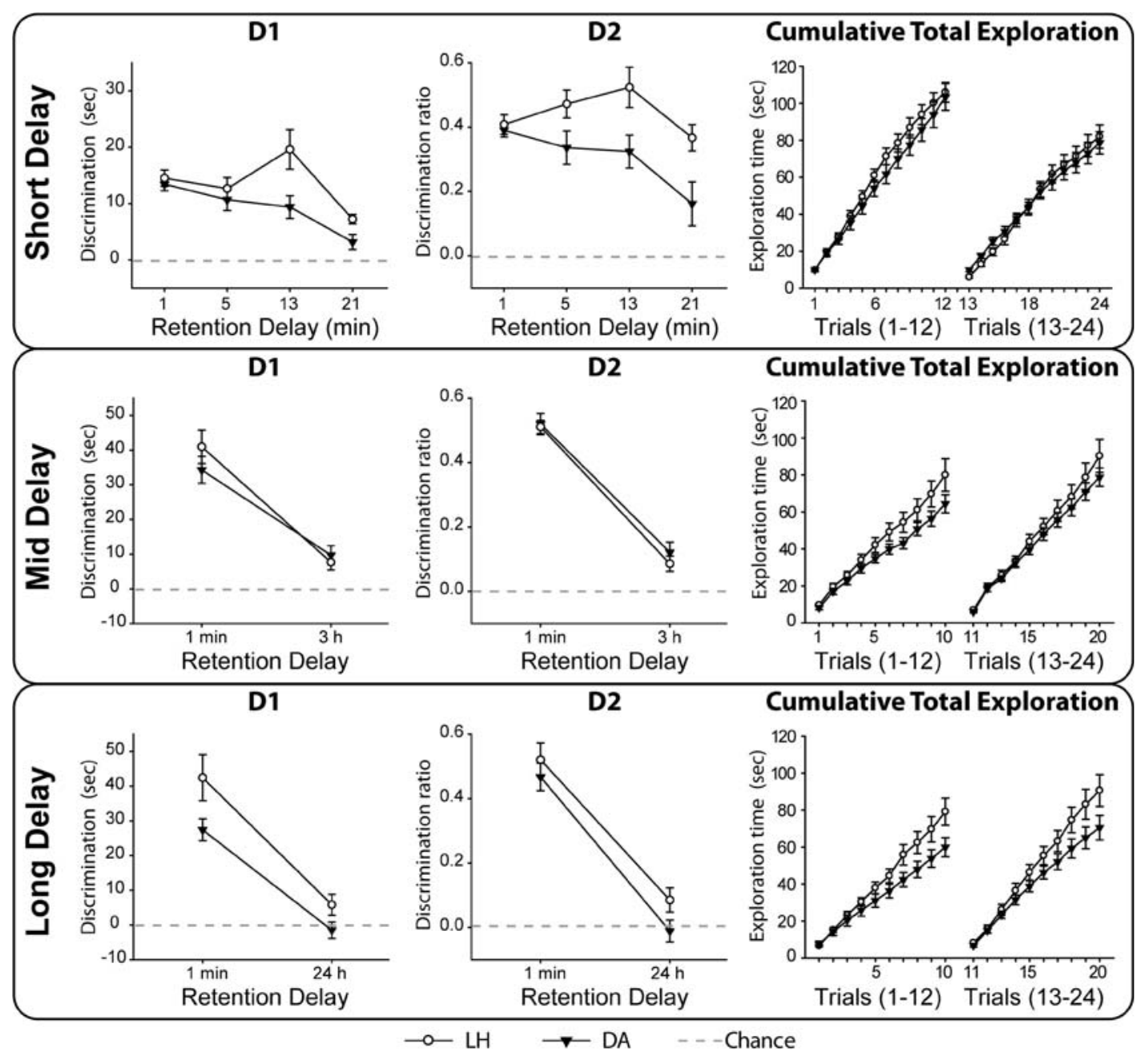

Figure 3. Experiment 1, Stage 2: Object recognition with Short (top; 5-, 13-, 21-min), Mid (middle; 3-h), and Long (bottom; 24-h) retention delays. (Left) D1 score; (middle) D2 ratio; (right) total exploration for all objects. For the Short Delay, each mean D1 and D2 score is taken from a block of four trials. Retention intervals increased by $2 \mathrm{~min}$ for every trial after Trial 12. The data were, therefore, blocked into three groups of four trials (Trials $13-16,17-20,21-24)$, with respective mean retention intervals of 5,13 , and 21 min. For the Mid and Long delays, each mean D1 and D2 score is taken from 10 trials. (Black symbols) Dark Agouti rats (DA), (white symbols) Lister Hooded rats (LH). For D1 and D2 scores, a score of zero reflects a failure to discriminate novel from familiar (chance). (Vertical bars) Standard error of the mean (although when small, they are obscured by the symbols).

D1: LH, $t_{(11)}=3.45, \quad P=0.003 ;$ DA $, t_{(11)}=3.53, \quad P=0.003$ updated D2: LH, $t_{(11)}=3.60, P=0.002 ; \mathrm{DA}, t_{(11)}=3.89, P=$ $0.002)$. Total exploration times did not differ between strains $\left(F_{(1,22)}=2.67, P=0.12\right)$.

Retention delays of $24 \mathrm{~h}$ (Long Delay, 20 trials). Analyses of the 24-h retention delay used a one between-group (LH and DA strains) by one within-group $(<1 \mathrm{~min}$ and $24 \mathrm{~h})$ ANOVA. Comparisons for $<1$ min (Trials 1-10) versus $24 \mathrm{~h}$ (Trials 11-20; Table 1B) retention intervals confirmed the superior performance at the short delay (Fig. 3, bottom; D1: $F_{(1,22)}=51.91, P<0.001 ;$ D2: $F_{(1,22)}=$ 105.4, $P<0.001$ ). Lister Hooded rats had superior scores (D1: $F_{(1,22)}=9.14, P=0.006 ;$ D2: $\left.F_{(1,22)}=3.55, P=0.073\right)$, though there was no strain $\times$ delay interaction (D1: $F<1$; D2: $F<1$ ). After the 24-h delay, only LH rats significantly discriminated the novel objects (one-sample $t$-test; cumulative D1: LH, $t_{(11)}=1.94$, $P=0.040 ;$ DA $t_{(11)}=0.645, P=0.26$; updated D2 LH, $t_{(11)}=$ 2.27, $P=0.022$; DA $\left.t_{(11)}=0.33, P=0.37\right)$. The LH rats showed more total exploration (novel and familiar objects) than the DA rats $\left(F_{(1,22)}=4.44, P=0.047\right)$.

Stage 3: Improving levels of object recognition after a 3-h retention interval Rats were again tested on whether they could discriminate between a novel object and an object presented $3 \mathrm{~h}$ earlier. This time the rat was exposed to the familiar objects once ("single") or $6 \times$ ("repeated") during the sample phase (Table 1C). The discrimination scores and the total exploration in the delay phase (Table 1C) were analyzed using a one between-group (LH and DA strains) by one within-group ("single" and "repeated") ANOVA.

Delay phase. Overall, D1 scores were higher for "repeated" objects (Fig. 4; $\left.F_{(1,22)}=5.55, P=0.028\right)$, though both strains performed above chance for both "single" and "repeated" objects (onesample $t$-test; repeated objects: LH, $t_{(11)}=4.64, P<0.001$; DA, $t_{(11)}=4.11, P=0.001$; single objects: LH, $t_{(11)}=3.71, P=0.002$; DA, $\left.t_{(11)}=2.14, P=0.028\right)$. There was no strain difference (D1, 
D1

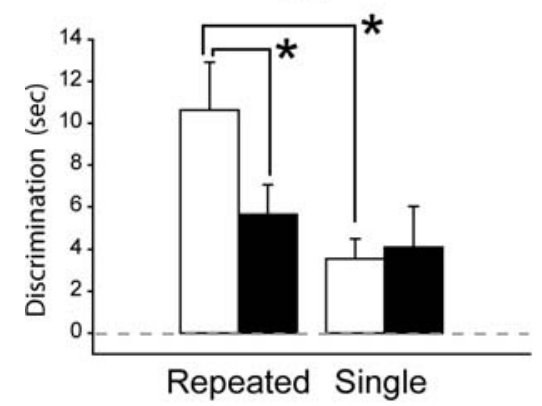

D2

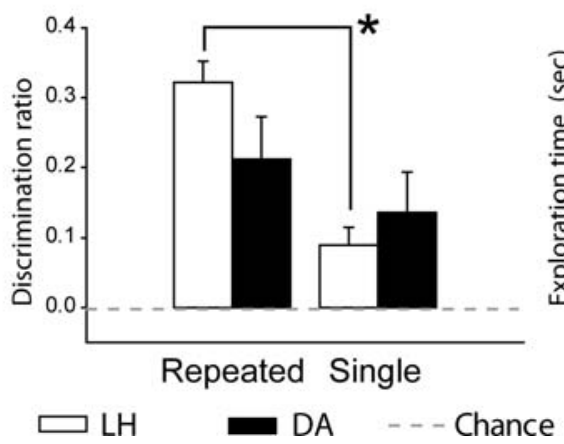

Total Exploration

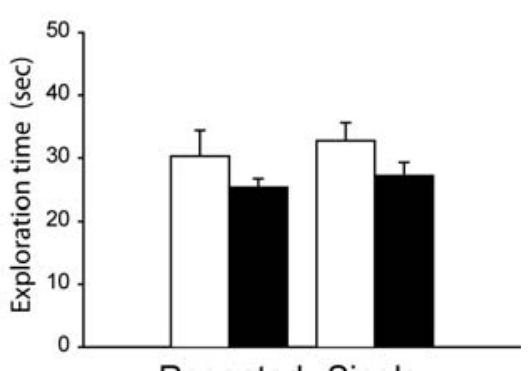

Repeated Single

Figure 4. Experiment 1, Stage 3: Improving levels of object recognition after a 3-h retention interval. The bar charts depict the mean performance of four trials for the "repeated" objects (presented six times) and four trials for the "single" objects (presented only once) in the delay phase. (Left) D1 score; (middle) D2 ratio; (right) total exploration for all objects. (Black bars) Dark Agouti rats (DA), (white bars) Lister Hooded rats (LH). For D1 and D2 scores, a score of zero reflects a failure to discriminate novel from familiar (chance). (Vertical bars) Standard error of the mean. Group differences: $\left({ }^{*}\right) P<0.05$.

$\left.F_{(1,22)}=1.93, P=0.18\right)$ and no strain $\times$ object repetition interaction $\left(F_{(1,22)}=2.25 P=0.15\right)$.

The D2 analyses closely matched the D1 findings (Fig. 4$)$, as there was a benefit of object repetition $\left(F_{(1,22)}=7.20, P=0.014\right)$ but no main effect of strain $\left(F_{(1,22)}=1.03, P=0.32\right)$ and no group $\times$ object repetition interaction $\left(F_{(1,22)}=1.88, P=0.18\right)$. Both strains performed above chance for both repeated and single object conditions (one-sample $t$-test; repeated objects: $\mathrm{LH}, t_{(11)}=$ 10.61, $P<0.001$; DA, $t_{(11)}=3.48, P=0.003$; single objects: $\mathrm{LH}$, $\left.t_{(11)}=3.49, P=0.003 ; \mathrm{DA}, t_{(11)}=2.40, P=0.018\right)$. Finally, the two strains did not differ in their overall exploration (novel + familiar objects) times $\left(F_{(1,22)}=2.25, P=0.15\right.$; Fig. 4$)$.

\section{Experiment 2: Standard object recognition-hippocampal and fornix lesions \\ Comparisons between the lesion and surgical control groups were analyzed with Student $t$-tests (two-tailed). Whether performances were above chance was determined using one-sample $t$-tests (one-tailed).}

\section{Hippocampal lesions (Lister Hooded rats)}

Histology. One rat was excluded as it had extensive sparing throughout CA1-3 and the dentate gyrus. In the remaining 10 cases, hippocampal damage (Fig. 5, upper panel) was most extensive in the dorsal parts of the structure. Analyses of the extent of the lesions in the hippocampus proper (dentate gyrus, CA1-4) revealed that four animals had $\geq 95 \%$ of the dorsal and $>50 \%$ of the ventral hippocampus damaged; three animals had $95 \%-70 \%$ of the dorsal and $70 \%-50 \%$ of the ventral hippocampus damaged; and three animals had $<70 \%$ of the dorsal (median 50\%) hippocampus damaged, of which two had $<10 \%$ tissue loss in the ventral part of the hippocampus. Overall, there was considerable damage to dorsal CA1 in all rats, though in three cases there was some sparing of the lateral parts of the dorsal dentate gyrus. In five cases, some sparing of the medial blade of the dentate gyrus was observed. The lateral dorsal CA3 region remained largely intact in four rats. Cell loss in the temporal hippocampus was most marked at caudal levels, with more appreciable sparing in the rostral parts of the dentate gyrus and CA1. In two rats, sparing of CA1 and the lateral blade of the dentate gyrus continued throughout the temporal hippocampus. The ventral subiculum was damaged in eight rats, but this damage was contained to the most rostral sections in six rats. Finally, there was frequent, but limited, damage to cortical areas dorsal to the hippocampus including the primary and secondary motor areas, the deep layers of the primary somatosensory area, the parietal region of the posterior association area, and parts of the primary and rostrolateral visual areas.

Behavior. All animals received a single session of 20 trials (retention $<1 \mathrm{~min}$ ). No behavioral differences were found between the

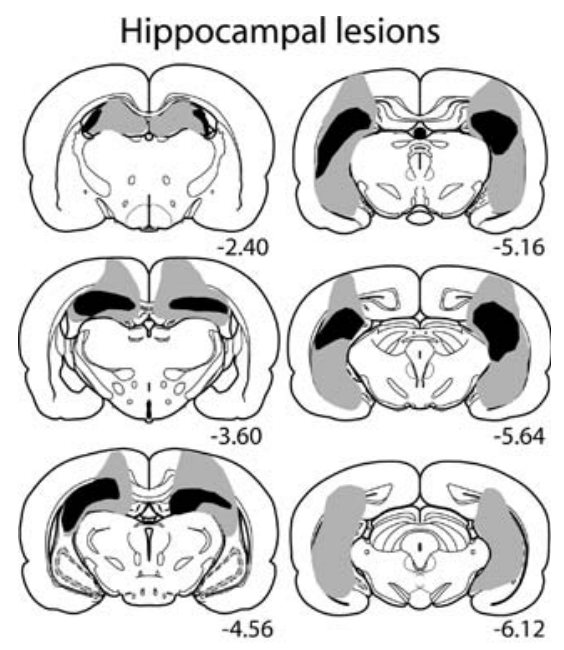

Fornix lesions

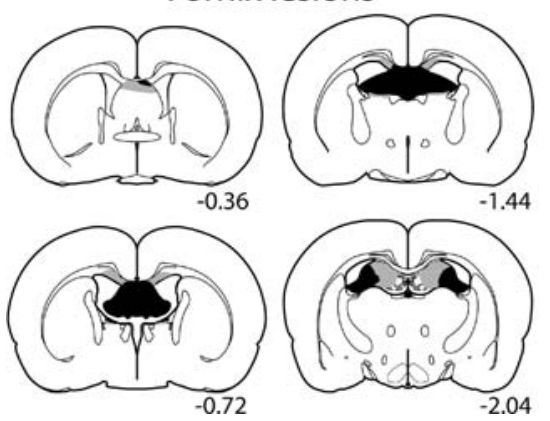

Figure 5. Diagrammatic reconstructions of the hippocampal (upper panel) and fornix (lower panel) lesions showing the cases with the largest (gray) and smallest (black) lesions. The numbers refer to the distance (in millimeters) from bregma (based on Paxinos and Watson 2005). 
hippocampal and sham groups (Fig. 6, top) for cumulative D1 $\left(t\right.$-test; $\left.t_{(19)}=0.35, P=0.727\right)$ or updated D2 ( $t$-test; $t_{(19)}=0.14$, $P=0.889$ ). Both groups preferred the novel objects (one-sample t-test; D1 sham group: $t_{(10)}=10.93, P<0.001$; D1 hippocampal group: $t_{(9)}=12.44, P<0.001$; D2 sham group: $t_{(10)}=15.91, P<$ 0.001; D2 hippocampal group: $\left.t_{(9)}=15.12, P<0.001\right)$. Finally, the two groups did not differ on total object exploration ( $t$-test; $\left.t_{(19)}=1.01, P=0.323\right)$.

\section{Fornix lesions (Dark Agouti rats)}

Histology. The fornix lesions severed all of the tract in both hemispheres in all 10 cases (Fig. 5, lower panel). The lesions extended rostrally to include the dorsal part of the caudal septum. In addition, the lesions often included the most dorsal part of the anterodorsal thalamic nucleus and anteroventral thalamic nucleus, and involved the most rostral part of the (septal) hippocampus. In all cases, there was damage to the corpus callosum at the level of the lesion. One animal had additional damage to the cingulum bundle, and so was removed from all analyses.

Behavior. Both groups distinguished the novel objects according to their cumulative D1 (one-sample $t$-test; sham group: $t_{(7)}=10.35$, $P<0.001$; fornix group: $\left.t_{(8)}=11.30, P<0.001\right)$ and updated D2 (one-sample $t$-test; sham group: $t_{(7)}=12.74, P<0.001$; fornix group: $\left.t_{(9)}=19.04, P<0.001\right)$ scores (Fig. 6 , bottom). There was no evidence of a lesion effect on these same measures ( $t$-test; D1 $\left.t_{(15)}=1.25, P=0.23 ; \mathrm{D} 2 t_{(15)}=0.70, P=0.50\right)$. The rats with fornix lesions did, however, show more total exploration than the surgical controls ( $t$-test; $t_{(15)}=2.28, P=0.036$ ).

\section{Experiment 3: Standard object recognition-“one well” versus "two wells"}

Two naïve cohorts of LH rats were trained on the object recognition test (each session, 10 trials, retention interval $<1 \mathrm{~min}$ ). For one group ("two wells") the procedure matched that in Experiment 1. For the other group ("one well"), food was placed by the middle of the far wall so that objects did not need to be pushed aside by the rats to access the food (see Materials and Methods). The two groups were compared with $t$-tests (twotailed). Whether performance was above chance was determined using one-sample $t$-tests (one-tailed).

Both groups spent more time exploring novel objects than familiar objects (Fig. 7, top; one-sample $t$-test; cumulative D1: "one well," $t_{(7)}=15.73, P<0.001$; "two wells," $t_{(7)}=16.69, P<$ 0.001; updated D2: "one well," $t_{(7)}=17.71, P<0.001$; "two wells," $\left.t_{(7)}=14.75, P<0.001\right)$, and by the end of the session there was no evidence of a difference between the two test conditions ( $t$-test; cumulative D1: $t_{(14)}=1.06, P=0.31$; updated D2: $t_{(14)}=$ $1.16, P=0.26)$. The "two wells" rats displayed more total object exploration ( $t$-test; cumulative total exploration: $t_{(14)}=2.51, P=$ $0.025)$, but this group difference presumably reflects the fact that only these rats were rewarded for touching both objects.

\section{Experiment 4: Standard object recognition-mice}

Using the "one well" object recognition procedure (see Experiment 3), it was found that mice (strain C57Bl/6) showed a clear preference for novel objects (Fig. 7, bottom; one-sample $t$-test; cumulative D1: $t_{(11)}=5.42, P<0.001$; updated D2: $t_{(11)}=$ 9.99, $P<0.001)$.

\section{Discussion}

The present study arose from the perceived need to devise new behavioral tests of object recognition for rats and mice that complement existing tests and address specific limitations in current protocols. A general goal was to assay a method (bow-tie maze) that might prove reliable and would provide multiple trials per session (see also Piterkin et al. 2008) and, as a consequence, reduce

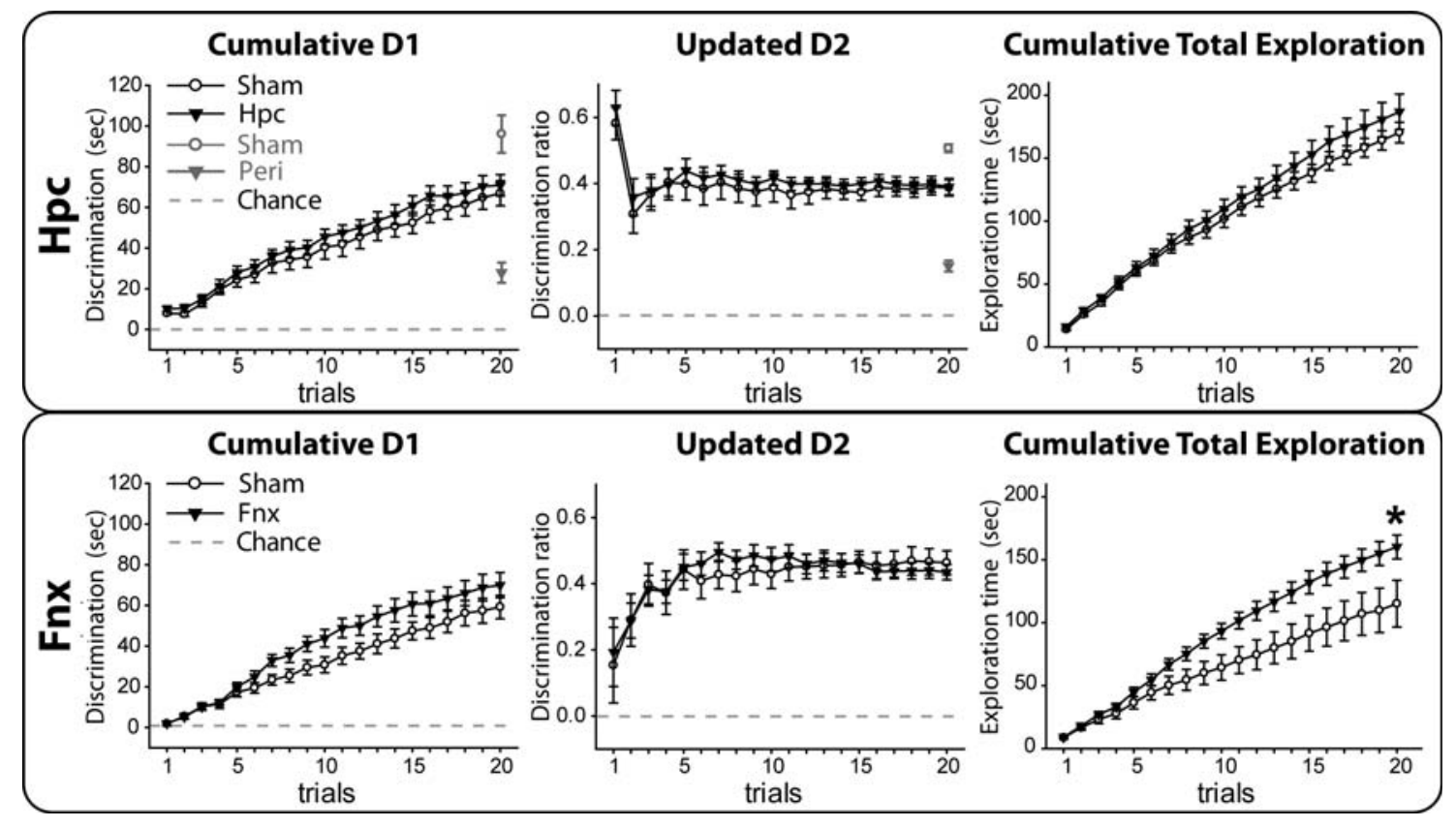

Figure 6. Experiment 2: Impact of hippocampal ( $\mathrm{Hpc}$, upper) and fornix (Fnx, lower) lesions on object recognition (retention interval $<1 \mathrm{~min})$. (Left) Cumulative D1 score, (middle) updated D2 ratio, (right) cumulative total exploration for all objects. (Black symbols) Performance of the lesioned animals, (white symbols) performance of the sham controls. For comparison purpose, the final D1 and D2 scores of rats with perirhinal lesions from Aggleton et al. (2010) have been added (gray symbols). For D1 and D2 scores, a score of zero reflects a failure to discriminate novel from familiar (chance). (Vertical bars) Standard error of the mean (although when small, they are obscured by the symbols). Group differences: $\left(^{*}\right) P<0.05$. 


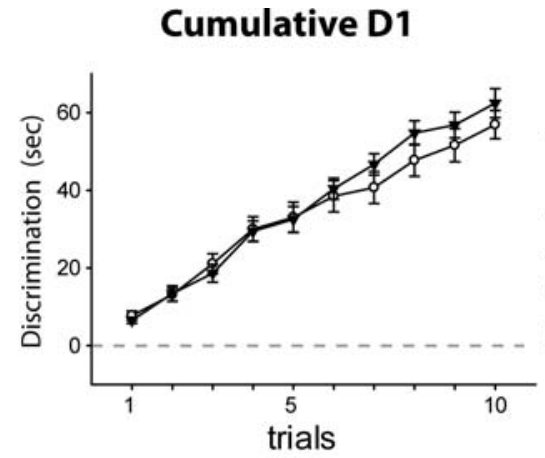

Cumulative D1

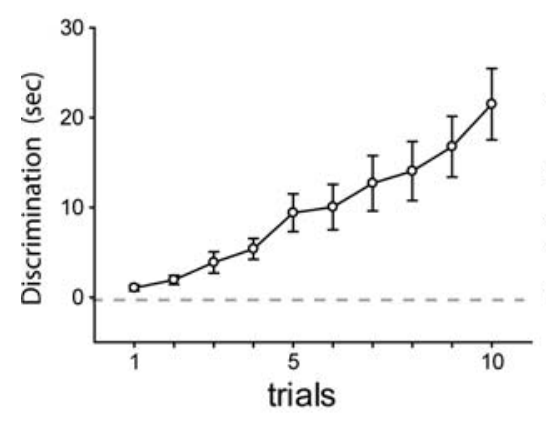

- $\mathrm{C} 57 \mathrm{Bl} / 6$ Mice
Updated D2

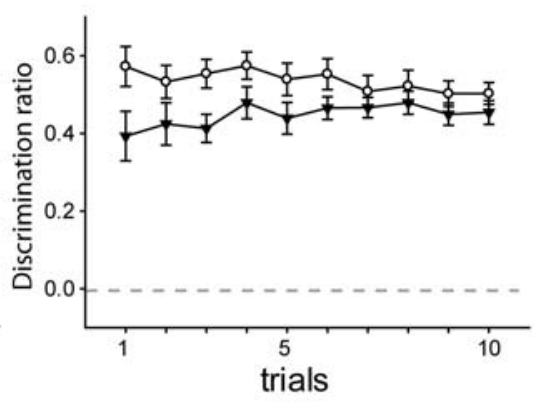

$\multimap 1$ well $\rightarrow-2$ wells --- Chance
Cumulative Total Exploration

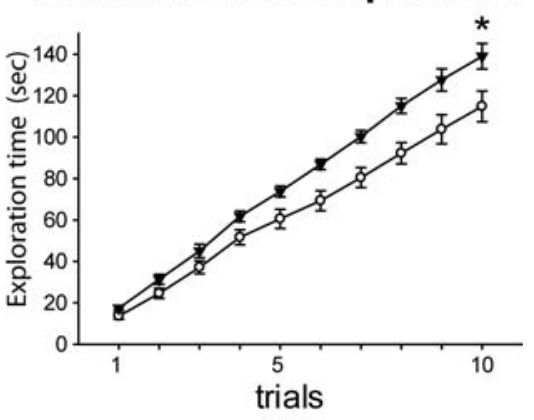

Cumulative Total Exploration
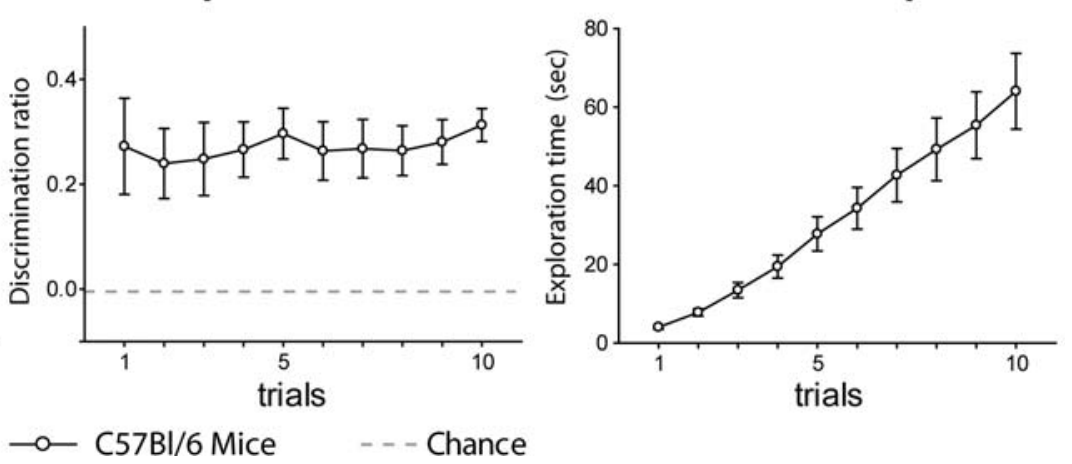

Figure 7. Experiments 3 (upper) and 4 (lower). (Upper) Mean performance in Experiment 3 of the "one-well" (white) group and the "two-wells" (black) group. (Lower) Performance of C57BI/6 mice (Experiment 4). (Left) Cumulative D1 score; (middle) updated D2 ratio; (right) cumulative total exploration for all objects. For D1 and D2 scores, a score of zero reflects a failure to discriminate novel from familiar (chance). (Vertical bars) Standard error of the mean (although when small, they are obscured by the symbols). Group differences: $\left(^{*}\right) P<0.05$.

much of the variance inherent in normal spontaneous recognition tasks. A more specific goal was, therefore, to test aspects of recognition not amenable to standard spontaneous tasks. One example was to examine forgetting curves based both on withinsession and across-session testing. In contrast to the standard spontaneous object recognition task, rats were rewarded in the bow-tie maze task for running back and forth as the objects were baited. However, as all objects are baited (both novel and familiar), these rewards do not guide discrimination performance. Indeed, Experiment 3 ("one well" versus "two wells") showed that even when the rewards were not directly underneath the objects ("one well"), animals still showed a strong preference for novelty. This "one well" design was then successfully adapted for mice, demonstrating the wider potential of this task, e.g., for transgenic animals.

The bow-tie maze consistently provided high levels of discrimination. Furthermore, because each object serves as both a novel and a familiar stimulus, the protocol counteracts the variance associated with individual object preferences. Experiment 1 (light) produced mean D1 scores ranging from 40-80 sec and mean D2 scores ranging from $0.3-0.4$ (depending on strain) for a single 20-min session (see also Aggleton et al. 2010; Albasser et al. 2010; Horne et al. 2010). These D1 are higher than those often achieved after combining two sessions to ensure side counterbalancing (also $\sim 20$ min of testing) in the standard spontaneous exploration task (D1 ranging from 10-20 sec in Ennaceur and Delacour [1988]; Dix and Aggleton [1999]). While the "updated" D2 scores in the present study were within the range of D2 scores reported in many spontaneous recognition studies (e.g., D2 of 0.4 in Ennaceur and Delacour [1988]; D2 of 0.4
Norman and Eacott [2004]; D2 of 0.2 in Dix and Aggleton [1999]), the small levels of variance created by summing multiple trials ensured more substantial effect sizes.

Taking advantage of the testing protocol, which involved baiting all objects, we were able to test object recognition in complete darkness. Both the LH and DA rats showed effective novelty discrimination in the dark, thereby supporting a recent study (Winters and Reid 2010) that reported effective tactile-based spontaneous object recognition. That study involved testing under red light (we used darkness), and their objects were not baited. Therefore, when vision is not available, rats may rely on other dimensions, such as odors, tactile information, and/or 3D representation to discriminate the objects. This assumption accords with the fact that rats cannot only discriminate novel from familiar odors (Fortin et al. 2004) and tactile textures (Birrell and Brown 2000), but can also perform tactile to visual cross-modal object recognition (Winters and Reid 2010). These findings also raise the possibility that tactile cues might contribute to the standard object recognition protocol, even though it is known that rats can solely rely on vision for recognition when required (Aggleton 1985; Prusky et al. 2004; Winters and Reid 2010).

Many previous studies have shown that object recognition performance by rats declines with increasing retention interval (Aggleton 1985; Ennaceur and Delacour 1988; Mumby and Pinel 1994). As far as we are aware, this is the first spontaneous object recognition study to examine this same effect within a single session. For retention delays of up to $24 \mathrm{~min}$, it was possible to test the rats without removing them from the apparatus. For longer delays, the session was divided by the retention interval ( $3 \mathrm{~h}$ or $24 \mathrm{~h})$. Summary forgetting curves were derived by combining 


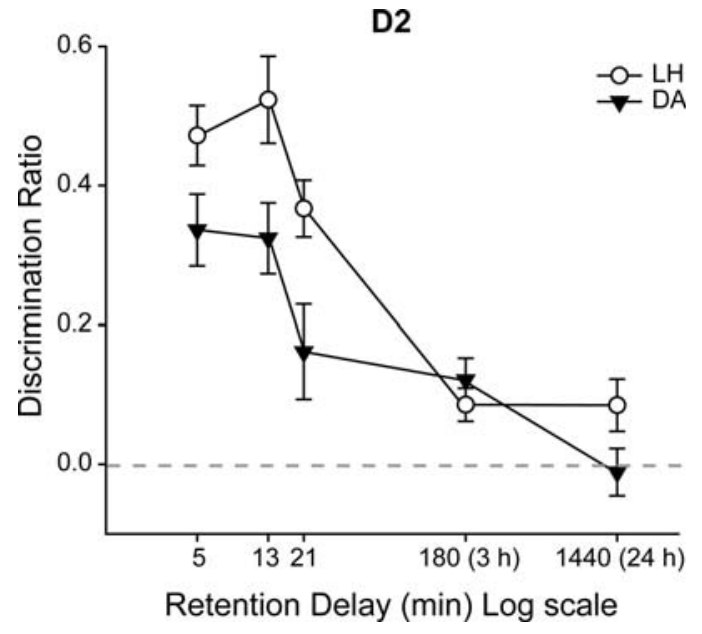

Figure 8. Summary of recognition performance across the delays used in Experiment 1, Stage 2. The graph depicts the mean performance (D2) of the rats, plotted against the retention interval (log). Performance declined with longer intervals. (Black symbols) Dark Agouti rats (DA), (white symbols) Lister Hooded rats (LH). A score of zero reflects a failure to discriminate novel from familiar (chance). (Vertical bars) Standard error of the mean.

both within-session and between-session data (Fig. 8). Only the Lister Hooded rats were still able to perform above chance after $24 \mathrm{~h}$ under these conditions. Even though other studies have found that object recognition memory can last for up to several weeks (Mumby et al. 2007; Broadbent et al. 2010), the present performance is notable because: (1) The familiarization phase was relatively short ( $<1 \mathrm{~min}),(2)$ the use of multiple trials within a session increases inter-item interference, yet performance remained above chance, and (3) the familiarization process provides a baseline level of object recognition (retention $<1 \mathrm{~min}$ ). The potential use of the bow-tie maze for the study of systemic or intracranial drug infusions provided the rationale for examining these different ways of testing the impact of delay on recognition. This same rationale explains why we also looked at performance after a 3-h delay with either single or multiple exposures of the familiar object (Experiment 1, Stage 3). The goal was to generate performance sufficiently above chance after $3 \mathrm{~h}$ so that it would be possible to identify an experimental manipulation that might attenuate performance at the same delay.

Even though the bow-tie maze offers many advantages (multiple trials, small variance), it is not without some disadvantages. The sample trials ( $1 \mathrm{~min}$ ) are shorter than those typically used in spontaneous object recognition, and this may partially explain the relatively poor levels of performance after a 3-h retention period (e.g., cf. Norman and Eacott 2004). An additional explanation is that the rats had to retain 10 objects rather than just one for $3 \mathrm{~h}$. Related to this additional memory load is the evidence from Experiment 1 (light) that 20 trials can lead to a build up of proactive interference, so that later trials are more demanding. Even so, novelty performance on the final trials is still effective as the cumulative D1 scores continue to rise (Fig. 2).

Throughout Experiment 1, two different rat strains (Lister Hooded and Dark Agouti) were compared. The Lister Hooded rats were quicker to train, and were superior whenever there was a group difference, e.g., first stage in the light, long delay. These strain differences cannot just be explained by the greater overall exploration of objects shown by LH rats, as the strain differences were found for both D1 and D2 (the latter measure adjusts for differences in total exploration). Previous studies of DA rats have shown that their visual acuity is similar to other pigmented rat strains but superior to albinos (Prusky et al. 2002; Burn 2008), while strain comparisons (DA, Sprague-Dawley, Fischer 344, PVG Hooded) using delayed nonmatching-to-sample showed them to be superior to these other rat strains (Aggleton 1996). In the present study, the inferior performance of the Dark Agouti rats compared with Lister Hooded is unlikely to be due to sensory differences, but might arise from their high levels of anxiety-like behavior (Mechan et al. 2002).

There is much debate over how temporal lobe structures interact to support the recognition of novel stimuli. Particular issues concern the relative roles of the perirhinal cortex and hippocampus (Aggleton and Brown 1999, 2006; Brown and Aggleton 2001; Eichenbaum et al. 2007; Squire et al. 2007). While lesions of the perirhinal cortex in animals (monkeys and rats) reliably disrupt visual recognition memory (Zola-Morgan et al. 1989; Meunier et al. 1993; Mumby and Pinel 1994; Ennaceur et al. 1996), the effects of hippocampal lesions remain uncertain (Winters et al. 2004, 2008; Forwood et al. 2005; Clark and Squire 2010). Previous studies using the same protocol in the bow-tie maze (Aggleton et al. 2010; Horne et al. 2010) found that perirhinal cortex lesions severely impair the standard task (retention $<1 \mathrm{~min}$ ). In contrast, the present study found that rats with hippocampal lesions, as well as rats with fornix lesions, can show normal levels of performance. Furthermore, overall levels of exploration following the hippocampal lesions were comparable to their controls, so removing a potential confound. While it seems evident that hippocampal lesions are not as disruptive as perirhinal cortex lesions at short delays, nothing further can be concluded without examining longer delays (Winters et al. 2004; Squire et al. 2007; Broadbent et al. 2010). Future experiments will, therefore, look at the impact of more complete hippocampal lesions on a wider array of different retention intervals. Finally, the lack of a deficit after fornix damage is consistent with previous rat studies using other tests of recognition memory (Shaw and Aggleton 1993; Ennaceur et al. 1997; Bussey et al. 2000).

The present study sought to extend the utility of the bow-tie maze, to examine object recognition in the dark, and to make preliminary observations on the impact of hippocampal system damage. It seems clear that the bow-tie maze task lends itself to a number of informative variants. It is also notable that normal rats continue to discriminate novel objects even after many hundreds of trials in which they were never food rewarded for first selecting the novel object. These findings show that the bow-tie maze can form the basis of a variety of recognition memory tasks for both rats and mice. As a consequence, it has the potential to provide a valuable complement to the standard spontaneous object recognition test.

\section{Materials and Methods}

\section{General methods, Experiments 1-4}

All experiments were performed in accordance with the UK Animals (Scientific Procedures) Act, 1986 and associated guidelines.

\section{Apparatus}

For Experiments 1, 2, and 3, rats were tested in a bow-tie-shaped maze made with opaque steel walls and a wooden floor (Fig. 1A). The maze was $120 \mathrm{~cm}$ long, $50 \mathrm{~cm}$ wide, and $50 \mathrm{~cm}$ high. Each end of the apparatus was triangular, the apices of which were joined by a narrow corridor $(12 \mathrm{~cm}$ wide). There was an opaque sliding door in the middle of the corridor that could be raised by the experimenter. The far wall of each triangle contained two recessed food wells, $3.5 \mathrm{~cm}$ in diameter and $2 \mathrm{~cm}$ 
deep (but see Experiment 3, Group "one well"). The food wells were separated by a short, opaque dividing wall that protruded $15 \mathrm{~cm}$ from the middle of the end wall. These food wells were covered by objects in the experiment proper.

\section{Objects}

Experiments 1-4 used numerous junk objects, each differing in shape, texture, size, and color (Supplemental Fig. 1). Every object was large enough to cover a food well but light enough to be displaced. Any object with an obvious scent was excluded. All objects had duplicates or triplicates so that identical objects could be used for different trials within a session. Specific objects were never repeated across sessions for a given animal. All objects were cleaned with alcohol wipes after each session.

\section{Object recognition-general protocol}

Each session contained multiple trials during which the animal could freely explore two objects, one novel and one familiar (Fig. 1B; Table 1A). To start each session, a rat was placed on one side of the maze, where a single object (object A) covered a food well that contained a single sucrose pellet ( $45 \mathrm{mg}$; Noyes Purified Rodent Diet). The rat remained in that part of the maze (with object A) for $1 \mathrm{~min}$. The central sliding door was then raised and the rat ran to the opposite side of the maze. There, the rat had a free choice between object A, now familiar, and novel object $B$ (Trial 1). Both objects A and B covered single sucrose pellets (except for Experiment 3-"one-well" condition and Experiment 4 -mice), and were concurrently available for the rat to explore for a total of $1 \mathrm{~min}$ (Fig. 1B). The sliding door was then raised (Trial 2) to reveal object B (now familiar) and object C (novel). This procedure continued; e.g., Trial 3 involved object $C$ (now familiar) versus object $\mathrm{D}$ (novel) so that 20 trials required 21 pairs of objects. Baiting of both the novel and familiar objects encouraged the animals' object exploration, but did not affect test validity as this relied on differential exploration. The placement of the novel object varied from left to right according to a pseudorandom schedule. In addition, the order of the particular objects used in the test was reversed for half of the rats. This counterbalancing ensured that the novel object in any given pair is reversed; e.g., for half of the rats in the trial B versus C, it is C that is novel (see above), but for the other half it is B that is novel.

\section{Analysis of behavior}

Animals were video-recorded throughout training. Object exploration was defined as directing the nose at a distance $<1 \mathrm{~cm}$ from the object, with the vibrissae moving, and/or touching it with the nose or the paws. Object exploration was not scored when animals sat on the object and when rats used the object to rear upward with the nose of the rat facing the ceiling. The duration of exploration was determined by holding down a key pad on a computer during the bursts of exploration recorded on video. Two discrimination measures were calculated (Ennaceur and Delacour 1988). D1 is the duration of exploration time devoted to the novel object minus the time devoted to the familiar object. Thus, the "cumulative D1" is the sum of the exploration times devoted to the novel objects across all trials minus the sum of the exploration times for the familiar objects. The second measure (D2) uses the difference in exploration times (i.e., D1) but then divides D1 by the total duration of exploration given to both the novel and familiar objects. The resulting D2 ratio can vary between +1 and -1 , with a positive ratio showing a preference for novel objects and a ratio of zero corresponding to no preference. The "updated D2" was the D2 ratio recalculated after each trial of the session.

\section{Statistical analysis}

One-sample $t$-tests were conducted using the cumulative data (D1 and D2) from final test trials to determine if animals performed above chance (zero), i.e., showed a preference for novelty
(Experiments 1-4). These one-sample $t$-tests were one-tailed as the only issue was whether scores were above chance. All remaining $t$-tests (e.g., between strains or lesion groups) were two-tailed. For Experiment 1 (Stages 1 and 2), the discrimination scores (D1 or D2) for the two rat strains were analyzed using a one betweensubject (groups) $\times$ one within-subject (conditions; light and dark, delays) ANOVA. When significant interactions were found, the simple effects for each condition were analyzed as recommended by Winer (1971) using the pooled error term; on occasions when there was a significant main effect but no interaction, the simple effects were examined so that regions that differed significantly between groups could be identified (Howell 1987).

\section{Experiment 1: Lister Hooded (LH) rats versus Dark Agouti (DA) rats}

\section{Animals}

Twelve naïve male, Lister Hooded rats (Harlan) and 12 male, Dark Agouti rats (Harlan) were used in Experiment 1. All rats were housed in pairs under diurnal conditions (14 h light/10 h dark), and water was provided ad libitum throughout the study. Animals were food-deprived up to $85 \%$ of their free-feeding body weight and were maintained above this level during the behavioral testing. Rats were $3 \mathrm{mo}$ old at the start of the study.

\section{Pretraining}

By the end of pretaining ( $7 \mathrm{~d}$ ), all Lister Hooded rats would run from one side of the maze to the other and displace an object covering a food well in order to reach food rewards. On day 1, pairs of rats were initially placed in the apparatus for $20 \mathrm{~min}$, where they explored the maze freely and ate sucrose pellets scattered on the floor and in the food wells ( $45 \mathrm{mg}$, Noyes Rodent Diet). On day 2 , animals were placed in the maze singly for $10 \mathrm{~min}$ where only the food wells were baited. From day 3, a single sucrose pellet was placed in each well and individual rats were rewarded for shuttling between the two goal areas, i.e., wells were constantly rebaited for $10 \mathrm{~min}$. The central sliding door was used to control movement from one side of the maze to the other. Rats were then trained to displace objects covering the wells in order to access the food reward. Initially the objects covered only a third of the well, so the reward was still visible. Objects were then moved progressively above the well to eventually cover it completely. Pretraining was complete when rats would quickly shuttle between the two ends of the maze (i.e., as soon as the sliding door was raised), and when rats were confident enough to displace the objects. Four pairs of objects were used during the pretraining, but these objects were not used in any of the following experiments. The pretraining protocol for the Dark Agouti rats (Experiment 1) used the same steps but took twice as long to complete.

\section{Stage 1: Standard object recognition: Light versus dark (delay $<1 \mathrm{~min}$ )}

All rats received three sessions (light, dark, light). Each session contained 20 trials during which the rat was exposed to two objects (one novel, one familiar) on every trial (Table 1A). The testing protocol was identical to that described above (General Protocol), with a retention interval $<1 \mathrm{~min}$.

For the light session, illumination was provided by ceiling lights giving a mean light intensity of $581.01 \times$ in the center of the maze. For the dark session, all lights were switched off giving a light intensity of $0.11 \mathrm{l} \times$. The experimenter wore night vision googles (Productive Firm Dipol Ltd.) and the session was recorded with an infrared camera (Maplin) fixed directly above the maze.

\section{Stage 2: Object recognition: Retention delays of $1 \mathrm{~min}$ to $24 \mathrm{~h}$}

Retention delays of 1-24 min (Short Delays, 24 trials). Rats received one session of 24 trials, of which the first 12 trials matched the general protocol (retention $<1 \mathrm{~min}$ ). Trial 12 was followed by a blank trial, where the animals ran to the other side of the maze to get 
food rewards, but no object was present. On Trial 13, the delay phase began. For Trials 13-24, a novel object was always presented with a familiar object that had been used during Trials 1-12 (and so had been presented on two adjacent trials, first when novel and then when familiar; see Table 1B). The objects made familiar from Trials 1-12 then occurred in the reverse order for delay testing; e.g., if A was presented in Trial 1, then the copy of A was the last familiar object used in the delay phase, i.e., Trial 24 . In this way, the retention interval, or lag, increased from 2 to 24 min across trials, an increase of 2 min per trial from Trials 13-24.

Retention delays of $3 \mathrm{~h}$ (Mid Delay, 20 trials). The procedure was very similar to the Short Delay, but the session only contained 20 trials (Table 1B). At the end of the first 10 trials (general protocol, retention $<1 \mathrm{~min}$ ), the rats were placed back in their home cage for $3 \mathrm{~h}$. The delay phase then followed with 10 further trials. On each trial (11-20), a novel object was presented along with an object that had been presented $3 \mathrm{~h}$ earlier during Trials 1-10.

Retention delays of $24 \mathrm{~h}$ (Long Delay, 20 trials). This condition (20 trials) was identical to the 3-h retention delay, except that the session was interrupted by an interval of $24 \mathrm{~h}$ after the first 10 trials (Table 1B).

Stage 3: Improving levels of object recognition after a 3-h retention interval Rats received two sessions. Each session consisted of two phases: a sample phase of 14 trials and a delay phase of eight trials (Table 1C). The two phases were separated by a delay of $3 \mathrm{~h}$, during which the animals were returned to their home cage. During the sample phase, eight different objects were used. As seen in Table 1C, four of the objects were presented six times $(A, C, D$, $\mathrm{G})$, and the other four objects (B, E, F, H) were presented only once. On each test trial, two different objects were presented together. The delay phase was the same as for Stage 2 (Mid Delay), as a novel object always occurred with a familiar object $(\mathrm{A}-\mathrm{H})$ that had been presented $3 \mathrm{~h}$ earlier.

\section{Experiment 2: Standard object recognition-hippocampal and fornix lesions}

\begin{abstract}
Animals
Twenty male, Lister Hooded (LH) rats (Harlan) and 18 male, Dark Agouti (DA) rats (Harlan) were used in Experiment 2. At the time of surgery the rats weighed 291-362 g (LH) or 215-268 g (DA). Housing and feeding conditions matched Experiment 1 . All rats had been used in very different previous experiments. The rats with hippocampal lesions (LH) were impaired in finding a submerged platform in a water-maze (M Horne and J Pearce, pers. comm.) while those with fornix lesions (DA) showed a severe deficit on reinforced spatial alternation in a T-maze (Vann et al. 2010). The LH rats (hippocampus) and the DA rats (fornix) were, respectively, $11 \mathrm{mo}$ and 6 mo old.
\end{abstract}

\section{Surgery}

Hippocampal lesions (Lister Hooded rats). Eleven rats received hippocampal lesions (Hpc). Rats were first anesthetized using an isofluraneoxygen mix and then placed in a stereotaxic frame (Kopf Instruments), with the incisor bar set at $0 \mathrm{~mm}$. A sagittal incision was made in the scalp, and the skin retracted to expose the skull. A dorsal craniotomy was made directly above the target region and the dura cut to expose the cortex. The rats in group Hpc were infused with ibotenic acid (Biosearch Technologies; dissolved in phosphate-buffered saline [pH 7.4] to provide a solution with a concentration of $63 \mathrm{mM}$ ) through a $2-\mu \mathrm{L}$ Hamilton syringe held with a microinjector (Kopf Instruments, Model 5000). Fourteen infusions per hemisphere were made at an infusion rate of $0.10 \mu \mathrm{L} / \mathrm{min}$ and a diffusion time of $2 \mathrm{~min}$ (for coordinates and volume see Iordanova et al. [2009]). The Sham group $(n=11)$ received identical treatment except that the dura was repeatedly perforated with a 25-gauge Microlance3 needle (Becton Dickinson) but no solution was infused into the brain.

Fornix lesions (Dark Agouti rats). Animals were deeply anesthetized by intraperitoneal injection $(60 \mathrm{mg} / \mathrm{kg})$ of sodium pentobarbital and then placed in a stereotaxic headholder (David Kopf Instruments) with the nose bar at $+5.0 \mathrm{~mm}$. The scalp was then cut and retracted to expose the skull. The fornix lesions $(n=10)$ were made by radiofrequency using an RFG4-A Lesion Maker (Radionics). An electrode ( $0.7 \mathrm{~mm}$ tip length, $0.25 \mathrm{~mm}$ diameter) was lowered vertically, and at each site the tip temperature was raised to $75^{\circ} \mathrm{C}$ for $60 \mathrm{sec}$. The coordinates, relative to ear-bar zero, were: (1) $\mathrm{AP}+5.3 \mathrm{~mm}, \mathrm{LM} \pm 0.7 \mathrm{~mm}$; (2) $\mathrm{AP}+5.3 \mathrm{~mm}$, $\mathrm{LM} \pm 1.8 \mathrm{~mm}$; the depths, from the top of cortex, were $-3.7 \mathrm{~mm}$ and $-3.8 \mathrm{~mm}$, respectively. The surgical control rats (Sham, $n=8$ ) received exactly the surgical procedures as the fornix rats except that the electrode was only lowered $2.0 \mathrm{~mm}$ from the top of the cortex in order to avoid damage to tracts. No radiofrequency lesion was made.

At the completion of all surgeries, the skin was sutured together over the skull and antibiotic powder was applied to the wound (Acramide, Dales Pharmaceuticals). All animals received $5 \mathrm{~mL}$ of glucose saline subcutaneously and were placed in a heated box until they showed signs of recovery. Paracetamol (for pain relief) and sucrose were dissolved in the rats' drinking water for several days post-surgery.

\section{Histological procedures}

Hippocampal lesions. On completion of the behavioral testing, all rats received a lethal overdose of sodium pentobarbital $(60 \mathrm{mg} / \mathrm{kg}$, Euthatal, Rhone Merieux). Rats were transcardially perfused, first with $0.9 \%$ saline and then with $10.0 \%$ formal-saline. Their brains were extracted, post-fixed for $24 \mathrm{~h}$, and then transferred to $30.0 \%$ distilled water sucrose solution in which they remained for a further $24 \mathrm{~h}$. Subsequently all brains were frozen in a $-20^{\circ} \mathrm{C}$ cryostat and sectioned coronally. The $40-\mu \mathrm{m}$ sections were collected on gelatine-coated slides, left to dry in room temperature over $24 \mathrm{~h}$, and then stained with cresyl violet, a Nissl stain.

The amount of damage in the hippocampus proper was measured with the program Analysis^D (Soft-Imaging Systems, Olympus). First, the normal area of the hippocampus was measured from six sections corresponding to $-2.40,-3.60,-4.56$, $-5.16,-5.64,-6.12$ relative to bregma (Paxinos and Watson 2005 ) in a surgical control. Then, using the same protocol, the extent of the hippocampal damage was quantified for each animal with a hippocampal lesion.

Fornix lesions. Rats were transcardially perfused with $0.1 \mathrm{M}$ phosphate buffer saline (PBS) followed by $4 \%$ paraformaldehyde in $0.1 \mathrm{M}$ PBS (PFA). The brains were removed and post-fixed in PFA for $4 \mathrm{~h}$ and then transferred to $25 \%$ sucrose overnight at room temperature with rotation. Then, brain sections were cut at $40 \mu \mathrm{m}$ on a freezing microtome in the coronal plane. A one-in-three series of sections was mounted onto gelatine-coated slides and stained with cresyl violet.

\section{Apparatus, objects, habituation, and pretraining}

All were identical to Experiment 1 (light).

\section{Behavioral testing}

Both cohorts received a single session of 20 trials during which each rat was exposed to two objects (one novel, one familiar) on 
every trial. The testing procedure was identical to that described in the General Protocol (Table 1A); i.e., the retention interval was $<1 \mathrm{~min}$.

\section{Experiment 3: Standard object recognition—“one well” versus "two wells"}

\section{Animals}

Sixteen naïve male, Lister Hooded rats (Harlan) were used. The animals were randomly divided in two groups: "one well" ( $n=$ $8)$ and "two wells" $(n=8)$. Housing conditions and rat ages were identical to Experiment 1.

\section{Apparatus: The bow-tie maze}

For Group "two wells" the apparatus was unchanged, but for Group "one well" the short dividing wall from each far end of the maze was removed. A new well $(3.5 \mathrm{~cm}$ diameter and $1 \mathrm{~cm}$ deep) was set at the middle of the end wall.

\section{Pretraining}

Pretraining for Group "two wells" was identical to that described for Experiment 1 and lasted 7 d. For Group "one well," the rats were rewarded for running between the single wells at the far ends of the maze, but not to push objects aside.

\section{Behavioral testing}

Testing used the General Protocol (e.g., Experiment 1, Stage 1), except that all rats received a single session of only 10 trials (see Trials 1-10, Table 1A). For Group "two wells," a single sucrose pellet was placed under every object before each trial. For Group "one well," two sucrose pellets were placed in the solitary wells. During testing, every rat was exposed to two objects (one novel, one familiar) on every trial (Fig. 1B).

\section{Experiment 4: Standard object recognition-mice}

\section{Animals}

Twelve naïve male C57Bl/6 mice (School of Biosciences, Cardiff University) were used. Mice were housed in boxes containing five, five, and two mice under diurnal conditions (14 h light/ $10 \mathrm{~h}$ dark), and food was provided ad libitum throughout the study. Two days before habituation, mice were water-deprived. Consequently, mice had free access to water for $4 \mathrm{~h}$ (box of five mice) or $3 \mathrm{~h}$ (box of two mice) each day. Mice were 3 mo old at the beginning of the experiment.

\section{Apparatus: The bow-tie maze}

The bow-tie maze was made of white, opaque foam board, and the floor was covered in sawdust. The apparatus was $80 \mathrm{~cm}$ long, $60 \mathrm{~cm}$ wide, and $30 \mathrm{~cm}$ high. Each end of the apparatus was triangular, the apices of which were joined by a narrow corridor $(8 \mathrm{~cm}$ wide and $10 \mathrm{~cm}$ long). This corridor contained a black, opaque L-shaped barrier that could be removed by the experimenter to block or give access to the other half of the maze. As in Experiment 3 "one well," there was only one well, situated against the middle of the end wall, and there was no dividing wall.

\section{Pretraining}

Like Experiment 3, Group "one well," the mice were trained until they would run back and forth between the central wells at both ends of the maze. Habituation and pretraining took $8 \mathrm{~d}$ (10 min each day per animal).

\section{Behavior}

Object recognition used the General Protocol. All mice received a single session of 10 trials, during which the mouse was exposed to two objects (one novel, one familiar) on every trial (see Trials 110, Table 1A). A single drop of Condensed Milk solution (half condensed milk, half water) was placed in each food well.

\section{Acknowledgment}

This work was supported by the Wellcome Trust [WT087855].

\section{References}

Aggleton JP. 1985. One-trial object recognition by rats. QJ Exp Psychol 37B: $279-294$.

Aggleton JP. 1996. The ability of different strains of rats to acquire a visual nonmatching-to-sample task. Psychobiology 24: 44-48.

Aggleton JP, Brown MW. 1999. Episodic memory, amnesia, and the hippocampal-anterior thalamic axis. Behav Brain Sci 22: 425-444; discussion 444-489.

Aggleton JP, Brown MW. 2006. Interleaving brain systems for episodic and recognition memory. Trends Cogn Sci 10: 455-463.

Aggleton JP, Vann SD. 2004. Testing the importance of the retrosplenial navigation system: Lesion size but not strain matters: A reply to Harker and Whishaw. Neurosci Biobehav Rev 28: 525-531.

Aggleton JP, Albasser MM, Aggleton DJ, Poirier GL, Pearce JM. 2010. Lesions of the rat perirhinal cortex spare the acquisition of a complex configural visual discrimination yet impair object recognition. Behav Neurosci 124: 55-68.

Albasser MM, Poirier GL, Aggleton JP. 2010. Qualitatively different modes of perirhinal-hippocampal engagement when rats explore novel vs. familiar objects as revealed by c-Fos imaging. Eur J Neurosci 31: $134-147$.

Bartko SJ, Winters BD, Cowell RA, Saksida LM, Bussey TJ. 2007. Perirhinal cortex resolves feature ambiguity in configural object recognition and perceptual oddity tasks. Learn Mem 14: 821-832.

Birrell JM, Brown VJ. 2000. Medial frontal cortex mediates perceptual attentional set shifting in the rat. J Neurosci 20: 4320-4324.

Broadbent NJ, Gaskin S, Squire LR, Clark RE. 2010. Object recognition memory and the rodent hippocampus. Learn Mem 17: 5-11.

Brown MW, Aggleton JP. 2001. Recognition memory: What are the roles of the perirhinal cortex and hippocampus? Nat Rev Neurosci 2: 51-61.

Burn CC. 2008. What is it like to be a rat? Rat sensory perception and its implications for experimental design and rat welfare. Appl Anim Behav Sci 112: $1-32$.

Bussey TJ, Duck J, Muir JL, Aggleton JP. 2000. Distinct patterns of behavioural impairments resulting from fornix transection or neurotoxic lesions of the perirhinal and postrhinal cortices in the rat. Behav Brain Res 111: 187-202.

Clark RE, Squire LR. 2010. An animal model of recognition memory and medial temporal lobe amnesia: History and current issues. Neuropsychologia 48: 2234-2244.

Clark RE, Zola SM, Squire LR. 2000. Impaired recognition memory in rats after damage to the hippocampus. J Neurosci 20: $8853-8860$.

Dere E, Huston JP, De Souza Silva MA. 2005. Episodic-like memory in mice: Simultaneous assessment of object, place and temporal order memory. Brain Res Brain Res Protoc 16: 10-19.

Dix SL, Aggleton JP. 1999. Extending the spontaneous preference test of recognition: Evidence of object-location and object-context recognition. Behav Brain Res 99: 191-200.

Eichenbaum H, Yonelinas AP, Ranganath C. 2007. The medial temporal lobe and recognition memory. Annu Rev Neurosci 30: 123-152.

Ennaceur A, Delacour J. 1988. A new one-trial test for neurobiological studies of memory in rats. 1: Behavioral data. Behav Brain Res 31: 47-59.

Ennaceur A, Neave N, Aggleton JP. 1996. Neurotoxic lesions of the perirhinal cortex do not mimic the behavioural effects of fornix transection in the rat. Behav Brain Res 80: 9-25.

Ennaceur A, Neave N, Aggleton JP. 1997. Spontaneous object recognition and object location memory in rats: The effects of lesions in the cingulate cortices, the medial prefrontal cortex, the cingulum bundle and the fornix. Exp Brain Res 113: 509-519.

Fortin NJ, Wright SP, Eichenbaum H. 2004. Recollection-like memory retrieval in rats is dependent on the hippocampus. Nature 431: $188-191$.

Forwood SE, Winters BD, Bussey TJ. 2005. Hippocampal lesions that abolish spatial maze performance spare object recognition memory at delays of up to $48 \mathrm{~h}$. Hippocampus 15: 347-355.

Gaskin S, Tremblay A, Mumby DG. 2003. Retrograde and anterograde object recognition in rats with hippocampal lesions. Hippocampus 13: 962-969.

Good MA, Hale G, Staal V. 2007. Impaired “episodic-like” object memory in adult APPswe transgenic mice. Behav Neurosci 121: 443-448. 
Harker KT, Whishaw IQ. 2004. A reaffirmation of the retrosplenial contribution to rodent navigation: Reviewing the influences of lesion, strain, and task. Neurosci Biobehav Rev 28: 485-496.

Horne MR, Iordanova MD, Albasser MM, Aggleton JP, Honey RC, Pearce JM. 2010. The impact of lesions of the perirhinal cortex on conditional spatial discriminations. Behav Neurosci 124: 311-320.

Howell DC. 1987. Statistical methods for psychology. Duxberry Press, Belmont, CA.

Iordanova MD, Burnett DJ, Aggleton JP, Good M, Honey RC. 2009. The role of the hippocampus in mnemonic integration and retrieval: Complementary evidence from lesion and inactivation studies. Eur J Neurosci 30: 2177-2189.

Kesner RP, Gilbert PE, Barua LA. 2002. The role of the hippocampus in memory for the temporal order of a sequence of odors. Behav Neurosci 116: $286-290$.

Mechan AO, Moran PM, Elliott M, Young AJ, Joseph MH, Green R. 2002. A comparison between Dark Agouti and Sprague-Dawley rats in their behaviour on the elevated plus-maze, open-field apparatus and activity meters, and their response to diazepam. Psychopharmacology 159: $188-195$.

Meunier M, Bachevalier J, Mishkin M, Murray EA. 1993. Effects on visual recognition of combined and separate ablations of the entorhinal and perirhinal cortex in rhesus monkeys. J Neurosci 13: 5418-5432.

Mishkin M, Delacour J. 1975. An analysis of short-term visual memory in the monkey. I Exp Psychol Anim Behav Process 1: 326-334.

Mumby DG. 2001. Perspectives on object-recognition memory following hippocampal damage: Lessons from studies in rats. Behav Brain Res 127: $159-181$.

Mumby DG, Pinel JP. 1994. Rhinal cortex lesions and object recognition in rats. Behav Neurosci 108: 11-18.

Mumby DG, Pinel JPJ, Wood ER. 1990. Nonreccuring-items delayed nonmatching-to-sample in rats: A new paradigm for testing nonspatial working memory. Psychobiology 18: 321-326.

Mumby DG, Piterkin P, Lecluse V, Lehmann H. 2007. Perirhinal cortex damage and anterograde object-recognition in rats after long retention intervals. Behav Brain Res 185: 82-87.

Norman G, Eacott MJ. 2004. Impaired object recognition with increasing levels of feature ambiguity in rats with perirhinal cortex lesions. Behav Brain Res 148: 79-91.

Otto T, Eichenbaum H. 1992. Complementary roles of the orbital prefrontal cortex and the perirhinal-entorhinal cortices in an odor-guided delayed-nonmatching-to-sample task. Behav Neurosci 106: $762-775$.

Paxinos G, Watson C. 2005. The rat brain in stereotaxic coordinates, 5 th ed. Academic Press, San Diego, CA.
Piterkin P, Cole E, Cossette MP, Gaskin S, Mumby DG. 2008. A limited role for the hippocampus in the modulation of novel-object preference by contextual cues. Learn Mem 15: 785-791.

Prusky GT, Harker KT, Douglas RM, Whishaw IQ. 2002. Variation in visual acuity within pigmented, and between pigmented and albino rat strains. Behav Brain Res 136: 339-348.

Prusky GT, Douglas RM, Nelson L, Shabanpoor A, Sutherland RJ. 2004. Visual memory task for rats reveals an essential role for hippocampus and perirhinal cortex. Proc Natl Acad Sci 101: 5064-5068.

Shaw C, Aggleton JP. 1993. The effects of fornix and medial prefrontal lesions on delayed non-matching-to-sample by rats. Behav Brain Res 54: 91-102.

Squire LR, Wixted JT, Clark RE. 2007. Recognition memory and the medial temporal lobe: A new perspective. Nat Rev Neurosci 8: $872-883$.

Steckler T, Drinkenburg WH, Sahgal A, Aggleton JP. 1998. Recognition memory in rats-I. Concepts and classification. Prog Neurobiol 54: 289-311.

Vann SD, Erichsen JT, O’Mara SM, Aggleton JP. 2010. Selective disconnection of the hippocampal formation projections to the mammillary bodies produces only mild deficits on spatial memory tasks: Implications for fornix function. Hippocampus (in press) doi: 10.1002/hipo. 20796

Winer MP. 1971. Statistical principles in experimental design. McGraw-Hill, New York.

Winters BD, Reid JM. 2010. A distributed cortical representation underlies crossmodal object recognition in rats. J Neurosci 30: 6253-6261.

Winters BD, Forwood SE, Cowell RA, Saksida LM, Bussey TJ. 2004. Double dissociation between the effects of peri-postrhinal cortex and hippocampal lesions on tests of object recognition and spatial memory: Heterogeneity of function within the temporal lobe. J Neurosci 24: 5901-5908.

Winters BD, Saksida LM, Bussey TJ. 2008. Object recognition memory: Neurobiological mechanisms of encoding, consolidation and retrieval. Neurosci Biobehav Rev 32: 1055-1070.

Wolff M, Gibb SJ, Dalrymple-Alford JC. 2006. Beyond spatial memory: The anterior thalamus and memory for the temporal order of a sequence of odor cues. J Neurosci 26: 2907-2913.

Zola-Morgan S, Squire LR, Amaral DG, Suzuki WA. 1989. Lesions of perirhinal and parahippocampal cortex that spare the amygdala and hippocampal formation produce severe memory impairment. $J$ Neurosci 9: 4355-4370.

Received May 21, 2010; accepted in revised form June 23, 2010. 


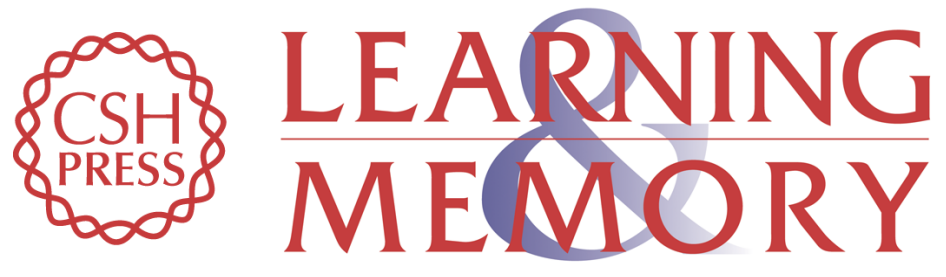

\section{New behavioral protocols to extend our knowledge of rodent object recognition memory}

Mathieu M. Albasser, Rosanna J. Chapman, Eman Amin, et al.

Learn. Mem. 2010, 17:

Access the most recent version at doi:10.1101//m.1879610

Supplemental
Material http://learnmem.cshlp.org/content/suppl/2010/08/03/17.8.407.DC1

References This article cites 50 articles, 11 of which can be accessed free at: http://learnmem.cshlp.org/content/17/8/407.full.html\#ref-list-1

License

Email Alerting Receive free email alerts when new articles cite this article - sign up in the box at the Service top right corner of the article or click here. 